\title{
PRESENTACIÓN DEL TEMA. Alfabetización en salud
}

\author{
Margarita R. Pino Juste ${ }^{1, a}$ \\ 1 Universidade de Vigo, España \\ a mpino@uvigo.es
}

Recibido: 22/09/2021; Aceptado: 06/10/2021

Existen diferentes definiciones y aproximaciones al concepto de alfabetización en salud (AS) (Finn y O’Fallon, 2017; Okan et al., 2019; Paakkari y Paakkari, 2019). Berkman et al. (2010) definen la AS como "el grado en el que los individuos tienen la capacidad para obtener, procesar y comprender la información y los servicios básicos de salud necesarios para tomar las decisiones que más se ajusten a sus necesidades". El Consorcio HLS-EU señala que la AS:

se basa en una alfabetización general e implica el conocimiento, la motivación y las competencias de las personas para acceder, comprender, evaluar y aplicar información de salud con el fin de emitir juicios y tomar decisiones en la vida cotidiana sobre atención médica, prevención de enfermedades, y promoción de la salud para mantener o mejorar la calidad de vida durante el curso de la vida (Sørensen et al., 2012).

Por tanto, podemos afirmar que la AS abarca diferentes habilidades y estrategias integradas que permiten mantener una buena salud (Mancuso, 2008).

Los principales componentes de la AS son el conocimiento teórico, el conocimiento práctico, el pensamiento crítico, la autoconciencia y la ciudadanía (Simovska et al., 2012).

Por tanto, la relación entre alfabetización en salud, promoción de la salud y educación para la salud es evidente. La promoción es el resultado de la educación para la salud (Tappe y Galer-Unti, 2001) y la AS es fruto de una adecuada educación y promoción de la salud.

Pero también existen algunas controversias con respecto al termino ya que algunos autores ponen de manifiesto que el concepto de AS supone solamente un reciclado de conceptos clásicos utilizados en promoción de la salud como "empoderamiento" o "la implicación del paciente" (Tones, 2002; Wills, 2009).

Sin embargo, la AS es un tema importante a considerar en la prestación de servicios de salud en todas las franjas etarias dados los desafíos de salud a los que nos enfrentamos. La alfabetización en salud (AS) condiciona la salud de las personas y la seguridad y calidad de la atención sanitaria que estas reciben (Pino-Juste, 2020). Por ejemplo, el rápido desarrollo de la enfermedad por coronavirus 2019 (COVID-19) en una pandemia ha exigido que las personas adquieran y apliquen información médica y adapten su comportamiento a un ritmo muy rápido (Paakkari y Okan, 2020) 
ya que el bajo nivel de conocimientos sobre salud de los adultos afecta en gran medida a su capacidad para utilizar la información sobre la salud y tomar decisiones coherentes sobre la salud de los hijos, de los educandos o de los pacientes, etc. (Berkman et al., 2011a; Bröder et al., 2017; Morrison et al., 2019). Las decisiones y conductas individuales afectan no solo de modo personal sino colectivo a toda la sociedad. Incluso para el cuidado de las personas mayores es necesaria una adecuada AS. No solo por la necesidad del autocuidado, sino también porque si el cuidador no tiene una adecuada AS puede verse afectado el cumplimiento del plan de atención médica para la persona mayor y está puede verse perjudicada inadvertidamente (Lindquist et al., 2011).

Según Morrison et al. (2019) el efecto de una baja alfabetización en salud influye en 1) conocimiento y conductas nutricionales deficientes, 2) tasas de obesidad más altas, 3) más errores de medicación, 4) mayor utilización de los servicios sanitario y emergencias y 5) conocimiento, conductas y resultados deficientes sobre algunas enfermedades como el asma.

Por tanto, el limitado nivel de AS representa un desafío importante para las políticas y prácticas sanitarias en toda Europa, aunque en un grado diferente según los distintos países (Sørensen et al., 2015).

A pesar de la demostrada importancia de la AS existe escasa investigación que proporcione información sobre el éxito de las diferentes intervenciones, sobre todo en el ámbito educativo (Berkman et al. 2011b; Schaffler et al., 2018; Fernández-Gutiérrez et al., 2018; Nutbeam et al., 2018). Ello se debe fundamentalmente a tres razones que señalan Visscher, et al. (2018): 1) las revisiones sistemáticas realizadas hasta el momento han demostrado que la AS inadecuada se asocia con peores resultados de salud, mayor uso y gasto de atención de la salud. 2) en todos los países europeos la población está envejeciendo y el número de personas con enfermedades crónicas está aumentando y una mejor AS en este grupo de edad puede ofrecer mayores oportunidades para participar activamente en la sociedad. 3) Y la mayoría de las investigaciones sobre AS se han realizado fuera de Europa.

En esta línea, con respecto a las estrategias educativas, Damián y Gallo (2020) proponen cuatro niveles de práctica: en organizaciones de atención médica, en asociaciones y colectivos comunitarios, aumentar las colaboraciones intersectoriales y colaborar con los proveedores de atención médica individuales.

Estas acciones se podrían complementar con campañas de información pública específicas y promoción de la AS en redes sociales para mejorar la calidad de la información identificando la desinformación y facilitando la toma de decisiones basadas en información valida y confiable (Okan et al., 2020).

Por otro lado, también es necesario que las administraciones públicas exijan y fomenten una formación en AS tanto de profesores como educadores y población en general.

Para dar respuesta a alguna de estas necesidades, en este monográfico se presentan cinco manuscritos que intentan aproximarse a la AS desde diferentes contextos sociales. El artículo "Alfabetizar en salud en la escuela" del Doctor de la Universidad de Valencia Valentín Gavidia Catalán, se aproxima a la evolución del concepto de salud e introduce la necesidad de que la escuela al alumnado en la toma de decisiones individual y colectiva.

En el artículo de los Doctores Anna Díaz-Vicario y Joaquín Gairín Sallán, de la Universidad Autónoma de Barcelona, sobre "La formación en salud y seguridad del profesorado para la gestión 
de centros educativos seguros y saludables", se debate la necesidad de contar con centros educativos seguros y saludables lo que requiere de la implicación de los equipos directivos y profesorado, pero también de recursos.

Por su parte, en el artículo de los Doctores de la Universidad de Vigo Millán Brea, Iago Portela y Mirian Alvariñas, se intenta dar respuesta a la pregunta “¿Influyen las variables sociodemográficas en el nivel de alfabetización en salud de los Monitores de Tiempo Libre en la Comunidad Autónoma de Galicia?", dado que los monitores de tiempo libre son agentes de salud que deben estar formados para transmitir este tipo de conocimientos.

El artículo sobre "Alfabetización en salud en la comunidad" de la Doctora Dolors Juvinyà-Canal, de la universidad de Girona, entiende la salud como un derecho y, por tanto, debe ser sostenida por toda la sociedad. Establece la alfabetización de la salud como un pilar básico de la promoción en salud, así como estrategias para facilitar la alfabetización en salud en la comunidad.

Por último, el artículo "Alfabetización en salud en el sistema sanitario: Objetivos y posibles actuaciones", que presenta la Dra. María Dolores Navarro-Rubio, de la Universidad Internacional de Catalunya, se centra en la necesidad de AS de los pacientes y sus cuidadores y el papel que deben adoptar los profesionales sanitarios y la propia organización sanitaria.

\section{REFERENCIAS BIBLIOGRÁFICAS}

Berkman, N. D., Davis, T. C. y McCormack, L. (2010). Health literacy: what is it? Journal of health communication, 15(S2), 9-19. https://doi.org/10.1080/10810730.2010.499985

Berkman, N. D., Sheridan, S. L., Donahue, K. E., Halpern, D. J. y Crotty, K. (2011a). Low health literacy and health outcomes: an updated systematic review. Annals of internal medicine, 155(2), 97-107. https://doi.org/10.1059/0003-4819-155-2-201107190-00005

Berkman, N. D., Sheridan, S. L., Donahue, K. E., Halpern, D. J., Viera, A., Crotty, K, et al. (2011b). Health literacy interventions and outcomes: An updated systematic review. Evidence report/technology assessment, (199), 1-941.

Bröder, J., Okan, O., Bauer, U., Bruland, D., Schlupp, S., Bollweg, T. M., ... y Pinheiro, P. (2017). Health literacy in childhood and youth: a systematic review of definitions and models. BMC public health, 17(1), 125. https://doi.org/10.1186/s12889-017-4267-y

Damian, A. J. y Gallo, J. J. (2020). Promoting health literacy during the COVID-19 pandemic: A call to action for healthcare professionals. The Harvard Kennedy School Misinformation Review, 1(3), https://doi.org/10.37016/mr-2020-027

Fernández-Gutiérrez, M., Bas-Sarmiento, P., Albar-Marín, M. J., Paloma-Castro, 0. y Romero-Sánchez, J. M. (2018). Health literacy interventions for immigrant populations: a systematic review. International Nursing Review, 65(1), 54-64. https://doi.org/10.1111/inr.12373

Finn, S. y O'Fallon, L. (2017). The emergence of environmental health literacy-from its roots to its future potential. Environmental health perspectives, 125(4), 495-501. https://doi.org/10.1289/ehp.1409337

Lindquist, L. A., Go, L., Fleisher, J., Jain, N., Friesema, E. y Baker, D. W. (2011). Relationship of health literacy to intentional and unintentional non-adherence of hospital discharge medications. Journal of General Internal Medicine, 27(2), 173-178. https://doi.org/10.1007/s11606-011-1886-3 
Mancuso, J. M. (2008). Health literacy: a concept/dimensional analysis. Nursing \& health sciences, 10(3), 248-255. https://doi.org/10.1111/j.1442-2018.2008.00394.x

Morrison, A. K., Glick, A. y Yin, H. S. (2019). Health literacy: implications for child health. Pediatrics in review, 40(6), 263-277. https://doi.org/10.1542/pir.2018-0027

Nutbeam, D., McGill, B. y Premkumar, P. (2018). Improving health literacy in community populations: a review of progress. Health promotion international, 33(5), 901-911. https://doi.org/10.1093/heapro/dax015

Orkan O., Bauer, U., Levin-Zamir, D., Pinheiro P. y Sørensen, K. (2019) (edit.). International Handbook of Health Literacy. Research, practice and policy across the lifespan. Policy Press.

Okan, O., Bollweg, T. M., Berens, E. M., Hurrelmann, K., Bauer, U. y Schaeffer, D. (2020). Coronavirus-related health literacy: A cross-sectional study in adults during the COVID-19 infodemic in Germany. International Journal of Environmental Research and public health, 17(15), 5503. https://doi.org/10.3390/ijerph17155503

Paakkari, L. y Okan, O. (2020). COVID-19: Health literacy is an underestimated problem. The Lancet. Public Health, 5(5), e249-e250. https://doi.org/10.1016/S2468-2667(20)30086-4

Paakkari, O. y Paakkari, L. (2019). Health literacy and the school curriculum: The example of Finland. En O. Okan, U. Bauer, D. Levin-Zamir, P. Pinheiro y K. Sørensen (edit.), International Handbook of Health Literacy Research, practice and policy across the lifespan (pp. 521-534). Policy Press. Recuperado de http://www.oapen.org/search?identifier=1005225

Pino-Juste, M. (2020). Alfabetización en Salud: Educar para Mejorar los Resultados en Salud. En M. C. Pérez Fuentes, et al. (comps). Intervención en Contextos Clínicos y de la Salud. Nuevas realidades (pp. 101110). Dykinson.

Schaffler, J., Leung, K., Tremblay, S., Merdsoy, L., Belzile, E., Lambrou, A. y Lambert, S. D. (2018). The effectiveness of self-management interventions for individuals with low health literacy and/or low income: a descriptive systematic review. Journal of general internal medicine, 33(4), 510-523. https://doi.org/10.1080/10810730.2011.604391

Simovska, V., Paakkari, L. y Paakkari, O. (2012). Health literacy as a learning outcome in schools. Health Education, 112(2), 133-152. https://doi.org/10.1108/09654281211203411

Sørensen K, Pelikan J. M, Röthlin F. (2015). Health literacy in Europe: comparative results of the European Health Literacy Survey (HLS-EU).Eur J Public Health, (25), 1053-1058. https://doi.org/10.1093/eurpub/ckv043

Sørensen, K., Van den Broucke, S., Fullam, J., Doyle, G., Pelikan, J., Slonska, Z., y Brand, H. (2012). Health literacy and public health: a systematic review and integration of definitions and models. BMC Public Health, 12(1), 80. https://doi.org/10.1186/1471-2458-12-80

Tappe, M. K. y Galer-Unti, R. A. (2001). Health educators' role in promoting health literacy and advocacy for the 21st century. Journal of School Health, 71(10), 477-482. https://doi.org/10.1111/i.17461561.2001.tb07284.x

Tones, K. (2002) Health literacy: new wine in old bottles? Health Education Research, 17(3), 287e-290. https://doi.org/10.1093/her/17.3.287

Visscher, B. B., Steunenberg, B., Heijmans, M., Hofstede, J. M., Devillé, W., van der Heide, I. y Rademakers, J. (2018). Evidence on the effectiveness of health literacy interventions in the EU: a systematic review. BMC public health, 18(1), 1-12. https://doi.org/10.1186/s12889-018-6331-7 
Wills, J. (2009). Health literacy: new packaging for health education or radical movement? International Journal of Public Health, 54(1), 3e-4. https://doi.org/10.1007/s00038-008-8141-7 